\title{
Maternal vitamin D status in pregnancy: a critical appraisal of current analytical data on maternal and neonatal outcomes
}

\author{
Spyridon N. Karras, ${ }^{1}$ Panagiotis Anagnostis, ${ }^{1}$ Andrea Petroczi, ${ }^{2}$ Cedric Annweiler, ${ }^{3,4}$ \\ Declan P. Naughton, ${ }^{2}$ Dimitrios G. Goulis ${ }^{1}$
}

${ }^{1}$ Unit of Reproductive Endocrinology, First Department of Obstetrics and Gynecology, Medical School, Aristotle University of Thessaloniki, Thessaloniki, Greece, ${ }^{2}$ School of Life Sciences, Kingston University, Kingston Upon Thames, London, United Kingdom, ${ }^{3}$ Department of Geriatric Medicine, UPRES EA 4638, University Hospital Angers, Angers, France; ${ }^{4}$ Robarts Research Institute, University of Western Ontario London, Ontario, Canada

\begin{abstract}
Maternal hypovitaminosis D during pregnancy has been associated with a wide spectrum of adverse maternal and neonatal health outcomes, some manifesting stronger associations than others. Research in this field has entered the "era of systematic data evaluation" via the conducting of a series of meta-analyses in an attempt to synthesize the diverse data from observational and supplementation studies. The aim of this review was to provide a critical appraisal of published observational and interventional (supplementation) meta-analyses on the link between maternal vitamin $D$ status and health consequences to both mothers and neonates. A literature review was performed by two reviewers in the Medline and Embase databases, from inception to March 2014, without any language restriction. Additional articles were identified by a manual search of the references from the key articles retrieved. The results provided evidence that the meta-analyses on the link between maternal vitamin D status and maternal / neonatal health consequences are characterized by a wide heterogeneity of studied populations as well as methodological pitfalls, including the absence of standardized vitamin $D$ assays and evaluation of vital external regulators of vitamin D bio-networks. Based on the above, interpretation of these meta-analyses should be carried out with care. Future supplementation studies should take into consideration all these population and methodological issues by incorporating them in their research design and settings.
\end{abstract}

Key words: Complications, Maternal, Meta-analysis, Neonate, Vitamin D deficiency, Vitamin D insufficiency, Pregnancy, Supplementation 


\section{BACKGROUND}

Maternal vitamin D deficiency during pregnancy has been associated with a plethora of adverse maternal health outcomes, some manifesting stronger associations than others. ${ }^{1}$ Although there is a great distance from an association between these two bio-phenomena to a verdict of causation, emphasis should be placed on the consistency of these observations indicating significant adverse health consequences arising from maternal vitamin $\mathrm{D}$ deficiency. ${ }^{2}$

Accumulating data associate maternal vitamin D deficiency throughout pregnancy with adverse maternal health outcomes, including glucose intolerance and gestational diabetes mellitus (GDM), pre-eclampsia, preterm birth and high rates of caesarian section. ${ }^{1,2}$ Meanwhile, maternal hypovitaminosis D has also been implicated in the pathogenesis of several adverse neonatal outcomes, including impaired skeletal and neurocognitive development, small birth weight and increased rates of autoimmune diseases in early infancy and childhood, including asthma, wheezing and type 1 diabetes. ${ }^{3}$ Recently, these observations were gradually incorporated into systematic evaluations, a process that significantly improved the potential for a holistic view of all available data in this field. With the escalating number of study outcomes available in the literature on vitamin $\mathrm{D}$ in pregnancy, meta-analyses have become important sources of information about where relationships exist between vitamin D status in mothers and maternal and neonatal health. This process could be characterized as representing the new "era of systematic data evaluation" of the link between maternal hypovitaminosis $\mathrm{D}$ and adverse maternal and neonatal outcomes.

The present review aims to provide an overview of available systematic reviews and meta-analyses on both maternal and neonatal data, in conjunction with an insight into certain methodological aspects, in an attempt to expand our current knowledge on vitamin D supplementation during pregnancy.

\section{METHODS - DATA SOURCES}

A literature review was performed by two reviewers independently (SNK and PA) with the use of the Medline and Embase databases. The search was focused on meta-analyses published from inception to March 2014, without any language restriction. The following keywords were used: "vitamin D", "pregnancy", "maternal complications", "neonatal complications", "supplementation studies" and "observational studies". Related articles and links were searched. Additional articles were identified by a manual search of the references from the key articles retrieved. Only articles exploring maternal vitamin $\mathrm{D}$ status and maternal/neonatal health consequences were selected.

\section{MATERNAL OUTCOMES: SYSTEMATIC REVIEWS AND META-ANALYSES}

A detailed review of available epidemiological data, including supplementation and prospective observational studies with intake of vitamin D or maternal 25(OH)D concentrations, dealt with a wide range of maternal and neonatal parameters. ${ }^{4}$ The review excluded trials of "very low" quality; however, dosing schemes varied significantly across all supplementation studies. Pooled analysis indicated no significant association between supplementation and preterm birth or mean duration of gestation. However, observational data on this field indicated a longer duration of gestation in women with adequate $(>50 \mathrm{nmol} / \mathrm{l})$ vitamin D status. In terms of maternal outcomes and mortality, observational data analyzed in this review were heterogeneous. The authors used preliminary supplementation data by the Hollis et al study $^{5}$ in which different dosing regimens (400 IU, 2000 IU, 4000 IU) for multiple problems including pre-eclampsia and GDM showed no significant benefit in the intention-to-treat analysis.

A comprehensive systematic analysis on the association between vitamin D status and several pregnancy outcomes [GDM, pre-eclampsia, bacterial vaginosis and small for gestational age (SGA)] in 22,000 women was conducted by Aghajafari et al. ${ }^{6}$ The group analyzed existing observational data, excluding studies where sampling was conducted during or after delivery, and developed a data extraction form for key indicators of study quality, including study design, control groups, $25(\mathrm{OH}) \mathrm{D}$ thresholds, gestational age and baseline confounders for vitamin D studies [body mass index (BMI), skin colour, clothing, race, season and expo- 
sure to ultra-violet radiation B (UVB)]. In total, 31 studies were included which differed in their design, statistical analysis, reporting of confounders and reporting of effect measures. The authors conducted two meta-analyses on each field. The first analysis used a calculated and adjusted odds ratio (OR) for quantifying the association between 25(OH)D insufficiency and the outcome (GDM, pre-eclampsia, birth variables). Since the studies, by definition designed to examine vitamin D status, reported separate proportions of deficiency and insufficiency, the authors combined available data to categorize concentrations less than $75 \mathrm{nmol} / 1$ for pregnancy outcomes and less than 37.5 $\mathrm{nmol} / \mathrm{l}$ for birth variables. The second analysis pooled weighted mean differences (WMD) to estimate potential differences in mean $25(\mathrm{OH}) \mathrm{D}$ concentrations between women who developed complications and those who did not. Overall, pooled OR showed that insufficient serum concentrations of $25(\mathrm{OH}) \mathrm{D}$ are associated with GDM, pre-eclampsia and SGA infants, demonstrating a positive moderate association for each variable. More specifically, hypovitaminosis D was associated with GDM [overall OR 1.49, 95\% confidence interval (CI) 1.18-1.88], which increases after adjustment for confounders in three studies (OR $1.98,95 \%$ CI 1.23-3.23) in the first analysis, whereas the second analysis showed that women with GDM had significantly lower concentrations of $25(\mathrm{OH})$ $\mathrm{D}$ than controls [pooled weighted mean difference (WMD) -7.36 nmol/1]. For pre-eclampsia, overall OR associated with maternal vitamin $\mathrm{D}$ insufficiency was 1.79 (95\% CI 1.25-2.58). In this case, adjustment for confounders led to an OR of 1.51 (95\% CI 0.89-2.57), whereas women with pre-eclampsia manifested significantly lower concentrations of $25(\mathrm{OH}) \mathrm{D}$ (pooled WMD - $14.53 \mathrm{nmol} / \mathrm{l}$ ). For birth variables, overall OR showed a significant positive association of SGA with low 25(OH)D concentrations $(1.85,95 \%$ CI 1.52 2.26). Reported data on birth weight (four studies) indicated that lower $(<37.5 \mathrm{nmol} / \mathrm{l})$ maternal $25(\mathrm{OH})$ D concentrations led to lower birth weight (-130.92 g). The analysis showed no dose-response relation between low 25(OH)D and pregnancy outcomes. It is noteworthy that included observational studies differed markedly in the assessment of maternal nutrition (where available) and the type of assay for $25(\mathrm{OH}) \mathrm{D}$ used. Although this analysis could not infer causality based on the observational data included, it could constitute a reliable initial basis for future supplementation trials.

Similar associations were evident in another recent meta-analysis on maternal hypovitaminosis D and pre-eclampsia (OR 2.09, 95\% CI 1.50-2.90), GDM (OR 1.38, 95\% CI 1.12-1.70) and preterm birth (OR $1.13,95 \%$ CI $0.74-1.71) .^{7}$ Exclusive observational data on GDM and pre-eclampsia were the objective of three recent meta-analyses. ${ }^{8-10}$

On this basis, seven observational trials were considered eligible for a meta-analysis on the field of GDM including 2146 participants of whom 433 were diagnosed with GDM with the majority of samples collected in the last trimester of pregnancy. ${ }^{8}$ Overall, four studies demonstrated significant differences in maternal serum 25(OH)D between women with GDM and controls. Most women (49\%) with GDM were vitamin D deficient [serum 25(OH)D $<50 \mathrm{nmol} / 1$ ], whereas a combined OR of 1.16 (95\% CI 1.19-2.17) between GDM and maternal hypovitaminosis D was evident. Potential confounders included maternal obesity, maternal age as well as ethnicity, resulting in significant associations after adjustment. However, studies included in the analysis used different diagnostic criteria for GDM resulting in potential selection bias, different techniques of measurement and a significant heterogeneity among studies was observed ( $\left.\mathrm{I}^{2} 69 \%\right)$.

Pre-eclampsia and its association with maternal hypovitaminosis D was systematically addressed in an elegant analysis including 15 observational studies, of which eight were included in a meta-analysis. ${ }^{9}$ In the systematic analysis, four studies found no such associations, whereas four others showed a significant association of lower concentrations of maternal $25(\mathrm{OH}) \mathrm{D}$ with an increased risk of preeclampsia. The remainder included studies which assessed maternal concentrations of $1,25(\mathrm{OH}) \mathrm{D}$, which could not be considered as a reliable marker of maternal vitamin D status. Several points from the meta-analysis (eight studies) are of significant importance: a) confounders adjusted, criteria for pre-eclampsia diagnosis and hypovitaminosis $\mathrm{D}$ were different within studies, b) a significant association between maternal vitamin D deficiency and the risk 
of pre-eclampsia was found although with significant heterogeneity ( $\left.\mathrm{I}^{2} 52.7 \%\right)$, and $\left.\mathrm{c}\right)$ in the subgroup analysis of studies with threshold values of $25(\mathrm{OH})$ $\mathrm{D}<38 \mathrm{nmol} / 1$, no significant associations were found, whereas in those that defined deficiency as $25(\mathrm{OH})$ $\mathrm{D}<50 \mathrm{nmol} / \mathrm{l}$ a significant association was evident. Although these findings could comprise a theoretical basis for supplementation trials, analysis interpretation has to overcome a paradox: the phenomenon of a significant association of pre-eclampsia and hypovitaminosis $\mathrm{D}$ in pregnant women defined as $25(\mathrm{OH})$ D concentrations $<50 \mathrm{nmol} / \mathrm{l}$ ) rather than those with more pronounced deficiency [25(OH)D $<38 \mathrm{ng} / \mathrm{ml}$ ] as would be pathophysiologically assumed.

A systematic effort which focused on incorporating both observational and supplementation data on the field of pre-eclampsia and maternal hypovitaminosis $\mathrm{D}$ was recently conducted by Hypponen et al. ${ }^{10}$ The authors conducted a systematic review and metaanalysis of prospective observational trials including data from the two large-scale epidemiological studies (HCCSA and ALSPAC) as well as randomized controlled trials (RCTs) using vitamin D supplementation for pre-eclampsia prevention. In two large-scale, observational studies on vitamin D supplementation and pre-eclampsia risk, a 19\% reduction in OR of pre-eclampsia was found, although the average intake of vitamin D was low (200-430 IU/day) for the majority of pregnant women. The second type of study analyzed were nine (including ALSPAC) observational studies which prospectively evaluated maternal $25(\mathrm{OH}) \mathrm{D}$ concentrations and risk for preeclampsia development. Heterogeneity in vitamin D assays used, criteria for vitamin D insufficiency and trimester of serum sampling was evident. Combined analysis demonstrated that mothers with higher $25(\mathrm{OH})$ D concentrations (different criteria among studies) a decreased OR of developing pre-eclampsia was found compared to those with low concentrations of $25(\mathrm{OH})$ D $(0.52,95 \%$ CI $0.30-0.89)$. However, there was a high heterogeneity ( $\left.\mathrm{I}^{2} 60 \%\right)$ among studies analyzed and this association was not evident in studies outside the US (latitudes south of $45^{\circ} \mathrm{N}$ ). RCTs on vitamin D supplementation and pre-eclampsia were also analyzed. Of the four placebo-controlled RCTs included in the meta-analysis only one was blinded and used supplementation with $400 \mathrm{IU} /$ day (comparison group) and 2000 and $4000 \mathrm{IU} /$ day as treatment groups. The other studies used supplementation with 450 to 1000 IU of vitamin $\mathrm{D} /$ day in combination with calcium, vitamin A and multivitamins. Data comparison showed a reduction in OR of pre-eclampsia of $0.66(95 \% \mathrm{CI}$ 0.52 - 0.83). Although data analysis revealed an association between vitamin $\mathrm{D}$ and pre-eclampsia across different study types, the design and power of many of the trials included characterizes this association as not conclusive. Although there is a strong theoretical basis, founded on extensive data concerning immunological and placental mechanisms, for supporting an association between vitamin D status during pregnancy and its impact on hypertensive disorders, available studies differ considerable in their design as well as definition of vitamin D status, parameters that affect data interpretation.

\section{NEONATAL OUTCOMES: META-ANALYSES AND SYSTEMATIC REVIEWS}

The fetus is completely reliant on maternal vitamin D stores for the supply of $25(\mathrm{OH}) \mathrm{D}$, which crosses the placenta. ${ }^{3,11}$ For this reason and based on the skeletal and non-skeletal effects of vitamin $\mathrm{D}$, it has been proposed that in utero vitamin $\mathrm{D}$ environment may influence the offspring's health and development. Regarding neonatal birth weight, we found three meta-analyses of observational studies, published in the last two years. ${ }^{4,6,7}$ The first did not come to a clear conclusion in terms of vitamin D status during pregnancy and birth weight, perhaps due to different thresholds used for defining vitamin D deficiency or insufficiency, but the risk of SGA seemed to be higher in vitamin $\mathrm{D}$ deficient women. ${ }^{4}$ The second one showed that women with $25(\mathrm{OH}) \mathrm{D}$ concentrations $<50 \mathrm{nmol} / 1$ were at increased risk of SGA (OR 1.52, 95\% CI 1.08-2.15) compared with those with $>50 \mathrm{nmol} / 1 .{ }^{6}$ This risk was confirmed by the third meta-analysis and was slightly higher (OR $1.85,95 \%$ CI 1.52-2.26 for vitamin D insufficiency). Of note, the latter meta-analysis was adjusted for possible confounders, such as $25(\mathrm{OH}) \mathrm{D}$ thresholds, gestational age at sampling among the studies and study design. The association between SGA infants and 25(OH)D insufficiency remained significant. ${ }^{6}$

A recent meta-analysis by Andersen et $\mathrm{al}^{12}$ also 
included data from RCTs with birth and neonatal anthropometry as the main outcome. Vitamin D supplementation resulted in increased birth weight in one study, which used no placebo and reported no data on serum $25(\mathrm{OH}) \mathrm{D}$. A significant effect was not evident in five other studies. Of major interest, in five RCTs, cord 25(OH)D concentrations were between $18-45 \%$ lower than maternal serum $25(\mathrm{OH}) \mathrm{D}$ at delivery. This wide variation is representative of the inherent flaws of vitamin D assays used in older studies.

\section{THE ERA OF SYSTEMATIC REVIEWS: METHODOLOGICAL CONSIDERATIONS}

\subsection{General aspects}

Essentially, systematic reviews aim to synthesize multiple results from original available data by using strategies delimiting systematic errors. From the methodological point of view, rules governing the objective and systematic selection of the included studies and the employed statistical analyses of the extracted data are clearly defined. Including an explicit reference to the systematic review checklist, assessment of bias and heterogeneity are expected as minimum quality checks. Owing to the noted poor performance of Cochrane's Q index in assessing heterogeneity, the preferred index should be the $\mathrm{I}^{2}$ statistics, which is an estimate of the percentage differences in combined effect sizes due to genuine heterogeneity. As a general rule, a random-effect model (RE) is preferred over fixed-effect (FE) meta-analysis if the data are not homogeneous. The choice between $\mathrm{RE}$ and FE also has an effect on the interpretation of the data. When using FE, we assume that the pooled data represent the common effect that is shared by all studies, whereas in RE, the pooled summary is the average of the study-specific effects. Because of this, the comparison of results from FE and RE models can provide further insights into the data and is thus recommended. To assess publication bias owing to negative results (namely, negative results are less likely to reach public dissemination compared to the positive results), meta-analyses ideally should incorporate a quantitative assessment of publication bias using a fail-safe number representing the number of studies with null findings. The exclusion/inclusion of non-significant results has a potentially profound impact on small effect sizes. The other inclusion/ exclusion bias arises from the study sample size. Balanced meta-analyses incorporate studies of all sizes, which should be demonstrated with a funnel plot, potentially accompanied by the relevant statistical indices (e.g., Egger's linear regression, Beggs' rank correlation) statistically assessing the asymmetry of the plot. Owing to the poor power of these tests, and in the absence of better ones at the moment, omission of the statistical test for asymmetry is a generally accepted practice.

\subsection{A methodological overview of analytical data on maternal vitamin D status in pregnancy}

The number of recent meta-analyses specific to pregnancy outcomes link maternal vitamin D status to pregnancy and neonatal outcomes. ${ }^{4,6} \mathrm{~A}$ broad spectrum of adverse pregnancy outcomes, ${ }^{7}$ specifically the risk of $\mathrm{GDM}^{8}$ and pre-eclampsia, ${ }^{9,10}$ testify to the importance of evaluating maternal vitamin $\mathrm{D}$ status during pregnancy but also to the uncertainty that is still present in this field. Despite a myriad of empirical studies being built on the assumption that a healthy vitamin $\mathrm{D}$ level is imperative for a healthy outcome of the pregnancy, Conde-Agudelo et $\mathrm{al}^{13}$ found that among the wide array of biomarkers, vitamin D concentrations were not a strong marker for predicting intrauterine growth restriction. The latter is potentially due to the confounding factors such as lifestyle, inter-individual variations and measurements. Albeit not focusing on pregnancy, a meta-regression of the conversion rate of total vitamin D intake from habitual intake via diet (and supplements where used) to the resulting circulating $25(\mathrm{OH}) \mathrm{D}$ concentration greatly depends on inter-individual variations and latitude. ${ }^{14}$ Issues surrounding the measurements of vitamin $\mathrm{D}$ concentrations add an emerging new facet to vitamin D research. ${ }^{15}$ Given the unwavering interest in the relationship between vitamin D levels and healthy pregnancy, it is surprising that a systematic assessment of maternal diet and the adequacy of micronutrients during pregnancy omits vitamin $\mathrm{D}$ but includes a wide range of other vitamins and minerals. ${ }^{16}$

Table 1 summarizes the key aspects of quality assessments of the meta-analyses on vitamin $\mathrm{D}$ and pregnancy included in this review. Meta-analyses fol- 


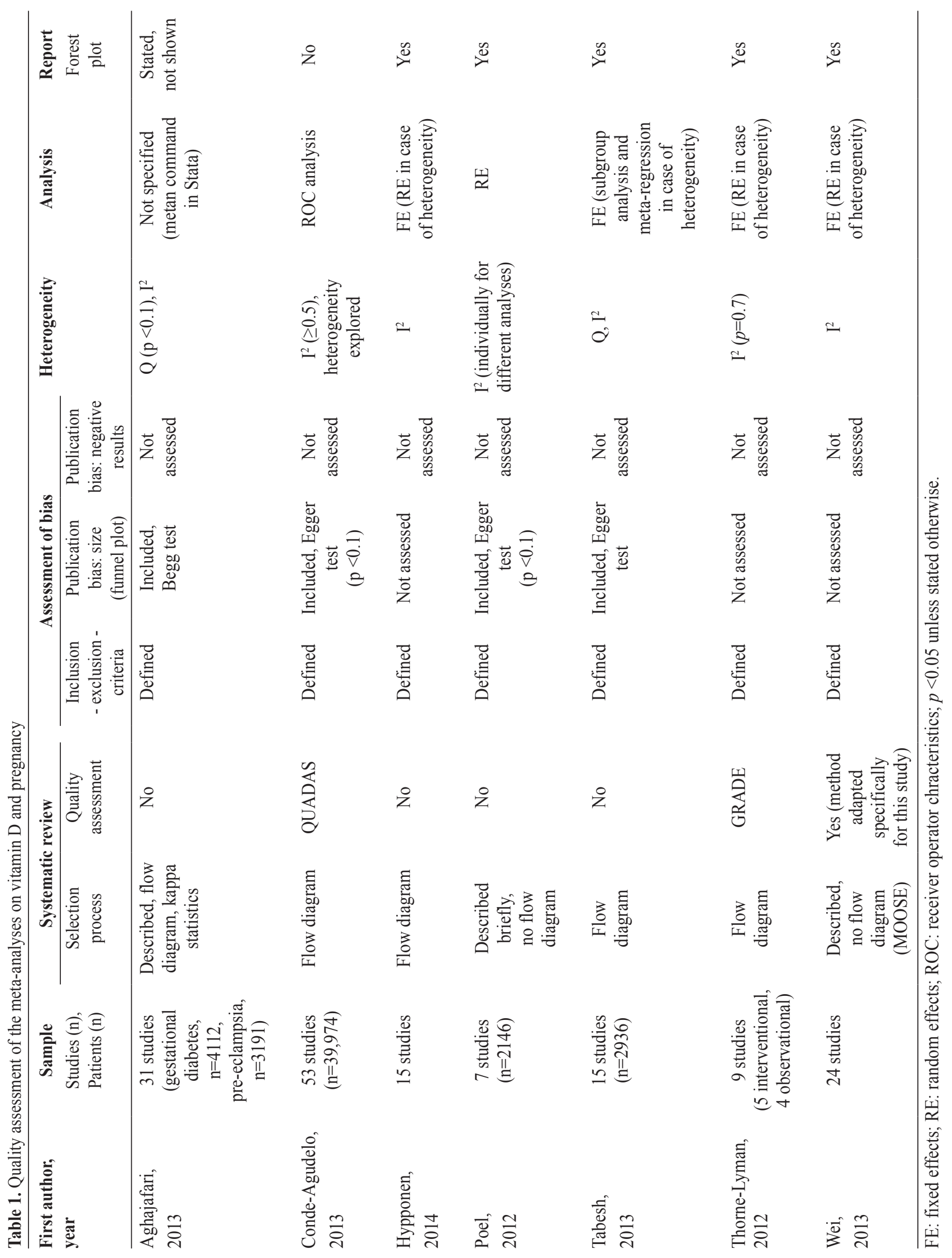


lowed the basic guideline for assessing heterogeneity to inform the statistical methods without exception. Bias assessment was limited to the funnel plot but publication bias was not considered. The approach to quality assessment varied across the included studies with several having no formal assessment of the quality. ${ }^{17,18}$ With one exception, information on the assay used to measure vitamin D concentrations was not included. The only study that included this information ${ }^{17}$ displayed six different methods in 24 studies. This point is especially pertinent as recent advances in analytical methodology have revealed that the measurements are far from facile for this complex set of vitamin $D$ analytes. Although it is beyond the aim of this review, it should be metioned that state-of-the art assays can measure up to 10 forms of vitamin D including epimers for the circulating forms $[25(\mathrm{OH})$ D]. ${ }^{19}$ The concurrent realization that different assays measure a variety of analytes adds considerable complexity to the extrapolation of previous studies to meta-analyses based on analyte levels.

\subsection{A critical evaluation of study characteristics included in systematic reports}

Among other health conditions, such as cardiovascular disease, cancer and neurodegenerative diseases, fertility and outcomes of pregnancy have been linked to vitamin $\mathrm{D}$ in an emphatic way. ${ }^{1,2}$ In the case of maternal hypovitaminosis $\mathrm{D}$ during pregnancy, this task manifests significant challenges, since a critical appraisal and synthesis of clinical findings are likely to be dependent on several exogenous parameters. Apart from the inherent flaws in the assay methodology used in most studies, a variety of additional factors affecting vitamin D status, including ultraviolet exposure, calcium intake or other clinical conditions that interfere with maternal and neonatal vitamin D status are key issues in the vitamin $\mathrm{D}$ equilibrium. ${ }^{20-23}$ These parameters were absent or only partially evaluated in most studies included in systematic reviews, despite their proven effects on population vitamin D status..$^{20-22}$ Moreover, vitamin D research issues per se incorporate unique characteristics including a geographical distribution and variation of individual vitamin D levels affected by local sartorial and social habits. In terms of dose supplementation, it could be stated that surely in the case of vitamin
$\mathrm{D}$, one dose does not fit all. ${ }^{24}$ We could not reach a conclusion on the beneficial effects of vitamin D on potential outcomes without a fair evaluation of the appropriate dose based on the vitamin D status of the population that will be supplemented. ${ }^{25}$ On this basis, it is evidently essential that vitamin D supplementation should not be directed only towards optimizing $25(\mathrm{OH}) \mathrm{D}$ concentrations, since this is not a condition, but rather to achieving a measurable improvement in a disease course. ${ }^{25}$ Certainly, this process requires a more in-depth knowledge of the global vitamin D status worldwide in conjunction with all reported aspects of vitamin D status reported previously.

\section{CONCLUSIONS}

It is clear that the new development in elucidation of the proposed associations between maternal vitamin $\mathrm{D}$ status and adverse pregnancy outcomes is the systematic evaluation of previous literature in the field. On that basis, systematic reviews indicate that maternal hypovitaminosis D is associated with a plethora of maternal complications. In our case, the thorough, systematic evaluation of previous studies in the field provided a more "clear-cut" view on the potential detrimental effects of maternal hypovitaminosis D, while it also underlined that the next step in the field would be carrying out of supplementation studies. At the same time however, it is evident that these systematic analyses are characterized by the wide heterogeneity of population samples included as well as methodological pitfalls, including the absence of standardized vitamin D assays and evaluation of vital external regulators of vitamin $\mathrm{D}$ bio-networks. By reason of the above, interpretation of these systematic analyses should be made carefully based on our current view of the so-called "vitamin D panacea" worldwide. In addition, future supplementation studies would benefit from the analyses conducted so far by incorporating the aforementioned issues in their research design and settings.

\section{DECLARATION OF INTEREST}

The authors declare that there is no conflict of interest that could be perceived as prejudicing the impartiality of the research reported. 


\section{REFERENCES}

1. Kovacs CS, 2008 Vitamin D in pregnancy and lactation: maternal, fetal, and neonatal outcomes from human and animal studies. Am J Clin Nutr 88: 520S-528S.

2. Anagnostis P, Karras S, Goulis DG, 2013 Vitamin D in human reproduction: a narrative review. Int J Clin Pract 67: 225-235.

3. Karras SN, Anagnostis P, Bili E, et al, 2014 Maternal vitamin $\mathrm{D}$ status in pregnancy and offspring bone development: the unmet needs of vitamin D era. Osteoporos Int 25: 795-805.

4. Thorne-Lyman A, Fawzi WW, 2012 Vitamin D during pregnancy and maternal, neonatal and infant health incomes: a systematic review and meta-analysis. Peadiatr Perinat Epidemiol 26: Suppl 1: 75-90.

5. Hollis BW, Johnson D, Hulsey TC, Ebeling M, Wagner CL, 2011 Vitamin D supplementation during pregnancy: double-blind, randomized clinical trial of safety and effectiveness. J Bone Miner Res 26: 2341-2357.

6. Aghajafari F, Nagulesapillai T, Ronksley PE, Tough SC, O'Beirne M, Rabi DM, 2013 Association between maternal serum 25-hydroxyvitamin D level and pregnancy and neonatal outcomes: systematic review and meta-analysis of observational studies. BMJ 346: f1169.

7. Wei SQ, Qi HP, Luo ZC, Fraser WD, 2013 Maternal vitamin D status and adverse pregnancy outcomes: a systematic review and meta-analysis. J Mat-Fet Neonat Med 26: 889-899.

8. Poel YHM, Hummel P, Lips P, Stam F, Van Der Ploeg T, Simsek S, 2012 Vitamin D and gestational diabetes: a systematic review and meta-analysis. Eur J Intern Med 23: 465-469.

9. Tabesh M, Salehi-Abargouei A, Tabesh M, Esmaillzadeh A, 2013 Maternal vitamin D status and risk of pre-eclampsia: a systematic review and meta-analysis. J Clin Endocrinol Metab 98: 3165-3173.

10. Hyppönen E, Cavadino A, Williams D, et al, 2013 Vitamin D and pre-eclampsia: original data, systematic review and meta-analysis. Ann Nutr Metab 63: 331-340.

11. Christesen HT, Elvander C, Lamont RF, Jørgensen JS, 2012 The impact of vitamin D in pregnancy on extraskeletal health in children: a systematic review. Acta Obstet Gynecol Scand 91: 1368-1380.

12. Karras SN, Shah I, Petroczi A, et al, 2013 An observational study reveals that neonatal vitamin $\mathrm{D}$ is primarily determined by maternal contributions: implications of a new assay on the roles of vitamin D forms. Nutr J 12: 77.

13. Conde-Agudelo A, Papageorghiou AT, Kennedy SH, Villar J, 2013 Novel biomarkers for predicting intrauterine growth restriction: a systematic review and meta-analysis. BJOG: 120: 681-694.

14. Cashman KD, Fitzgerald AP, Kiely M, Seamans KM, 2011 A systematic review and meta-regression analysis of the vitamin D intake-serum 25-hydroxyvitamin D relationship to inform European recommendations. $\mathrm{Br}$ J Nutr 106: 1638-1648.

15. Naughton DP, Petroczi A, 2014 Vitamin D status and ill health. Lancet Diabetes Endocrinol 22: 74-75.

16. Blumfield ML, Hure AJ, Macdonald-Wicks L, Smith R, Collins CE, 2013 Micronutrient intakes during pregnancy in developed countries: systematic review and meta-analysis. Nutr Rev 71: 118-132.

17. Haidich AB, 2010 Meta-analysis in medical research. Hippokratia 14: Suppl 1: 29-37.

18. Hunter JE, Schmidt FL. Methods of meta-analysis: correcting error and bias in research findings $\left(2^{\text {nd }} \mathrm{ed}\right)$, 2004, Thousand Oaks, CA: Sage.

19. Shah I, Petroczi A, Naughton DP, 2014 Exploring the role of vitamin $\mathrm{D}$ in type 1 diabetes, rheumatoid arthritis and Alzheimer's disease: new insights from accurate analyses of 10 forms. J Clin Endocrinol Metab 99: 808-816.

20. Hollis BW, Wagner CL, 2013 Vitamin D and pregnancy: skeletal effects, nonskeletal effects, and birth outcomes. Calcif Tissue Int 92: 128-139.

21. Holick MF, 1995 Environmental factors that influence the cutaneous production of vitamin D. Am J Clin Nutr 61: Suppl 3: 638-645.

22. Andersen LB, Abrahamsen B, Dalgård C, et al, 2013 Parity and tanned white skin as novel predictors of vitamin D status in early pregnancy: a population-based cohort study. Clin Endocrinol (Oxf) 79: 333-341.

23. Karras SN, Anagnostis P, Annweiler C, et al, 2014 Maternal vitamin D status during pregnancy: the Mediterranean reality. Eur J Clin Nutr 68: 864-869.

24. Karras SN, Anagnostis P, Beauchet O, Goulis DG, Annweiler C, 2014 Vitamin D supplements and bone mineral density. Lancet 383: 1292-1293.

25. Anagnostis P, Karras SN, Athyros VG, Annweiler C, Karagiannis A, 2014 The effect of vitamin D supplementation on skeletal, vascular, or cancer outcomes. Lancet Diabetes Endocrinol 2: 362-363. 